\section{A) Check for updates}

Cite this: Analyst, 2021, 146, 4010

\title{
Imaging mass spectrometry: a new way to distinguish dermal contact from administration of cocaine, using a single fingerprint $\dagger$
}

\author{
C. Costa, $t^{\mathrm{a}}$ M. Jang, $t^{\mathrm{b}}$ J. de Jesus, (D) ${ }^{\mathrm{c}}$ R. T. Steven, (D) ${ }^{\mathrm{d}}$ C. J. Nikula, (D) ${ }^{\mathrm{d}}$ E. Elia, ${ }^{d}$ \\ J. Bunch, ${ }^{d}$ A. T. Bellew, ${ }^{e}$ J. F. Watts, ${ }^{f}$ S. Hinder ${ }^{f}$ and M. J. Bailey (D) $* c$
}

\begin{abstract}
Here we show a new and significant application area for mass spectrometry imaging. The potential for fingerprints to reveal drug use has been widely reported, with potential applications in forensics and workplace drug testing. However, one unsolved issue is the inability to distinguish between drug administration and contamination by contact. Previous work using bulk mass spectrometry analysis has shown that this distinction can only be definitively made if the hands are washed prior to sample collection. Here, we illustrate how three mass spectrometry imaging approaches, desorption electrospray ionisation (DESI), matrix assisted laser desorption ionisation (MALDI) and time of flight secondary ion mass spectrometry (ToF-SIMS) can be used to visualise fingerprints at different pixel sizes, ranging from the whole fingerprint down to the pore structure. We show how each of these magnification scales can be used to distinguish between cocaine use and contact. We also demonstrate the first application of water cluster SIMS to a fingerprint sample, which was the sole method tested here that was capable of detecting excreted drug metabolites in fingerprints, while providing spatial resolution sufficient to resolve individual pore structure. We show that after administration of cocaine, lipids and salts in the fingerprint ridges spatially correlate with the cocaine metabolite, benzoylecgonine. In contrast after contact, we have observed that cocaine and its metabolite show a poor spatial correlation with the flow of the ridges.
\end{abstract}

Received 5th February 2021 Accepted 13th May 2021

DOI: $10.1039 / \mathrm{d} 1 \mathrm{an} 00232 \mathrm{e}$ rsc.li/analyst that as of 2018 approximately 5.3\% (or 269 million people) of the global population aged 15-64 had used illicit drugs in the previous year. There are an estimated 19 million cocaine users globally, and an all-time high production of cocaine. With this increase in illicit drug consumption and production, there is increasing interest to gain forensic intelligence on drug distribution and drug use.

Several groups have observed that illicit drugs can be detected in fingerprint residues, ${ }^{1-5,16-24}$ leading to questions about the circumstances of deposition. For example, what does it mean to detect an illicit drug in a fingerprint? Is the drug detected because of drug administration or due to dermal contact?

Recent work has focussed on the possibility of using a fingerprint for illicit drug testing, because a fingerprint offers a traceable and non-invasive approach compared with other sampling matrices. In drug testing, a fingerprint can be deposited under controlled conditions - for example the donor can be asked to wash their hands prior to deposition, and the deposition pressure, time and substrate can all be tailored to suit the application. This stands in contrast to forensic evidence, where for a fingerprint deposited at a crime scene, the circumstances of deposition are largely unknown. 
Jang et al. determined the mass of cocaine and its primary metabolite, benzoylecgonine (BZE) in fingerprints produced after dermal contact, as well as administration of cocaine. ${ }^{5}$ It was found that if hands are washed prior to sample collection, the cocaine entry route (contact/administration) could be determined based on the presence of BZE, which was only detected after administration. However, for unwashed hands, the total content of cocaine and BZE could not be used to determine the cocaine entry route. This observation has implications for the forensic interpretation of the detection of an illicit drug in a fingerprint at a crime scene. In forensic scenarios, a donor is unlikely to wash their hands prior to depositing a fingerprint. Therefore, methods based on bulk analysis, i.e. that measure only the mass of cocaine and metabolites deposited in a fingerprint, will be unable to determine whether the donor had used the illicit drug or simply encountered a contaminated surface.

To help overcome such issues, here we explore whether imaging of illicit drug residues in fingerprints may help distinguish drug use from drug contact. Mass spectrometry imaging tools have been previously applied to the detection of cocaine and its metabolites in fingerprints. ${ }^{15,17,21,22,25-27}$ They are attractive for forensic investigations, because unlike the bulk analysis approaches that have been developed for fingerprint drug testing, they do not destroy the sample. Ifa et al. showed that cocaine could be detected in a fingerprint after contact with cocaine powder using desorption electrospray ionisation (DESI) coupled to mass spectrometry. ${ }^{26}$ Bailey et al. showed that cocaine and its primary metabolite, benzoylecgonine, could be detected in fingerprints produced after ingestion of cocaine, using both DESI and matrix assisted laser desorption ionisation (MALDI). ${ }^{17}$ Whilst the feasibility of using these techniques for detecting drug residues in fingerprints has been demonstrated, spatial differences in drug residues have (to our knowledge) never been explored.

Fingerprints deposited at crime scenes are often invisible to the naked eye and must be developed for the ridges to be observed. Groeneveld et al. demonstrated that development of a fingerprint using cyanoacrylate fuming or vacuum metal deposition did not affect the detection of cocaine spiked into a fingerprint using MALDI. ${ }^{22}$ Similarly, Bradshaw et al. reported the detection of cocaine and the cocaine metabolite cocaethylene in a fingerprint that had been recovered from the interior of a window frame at a crime scene. ${ }^{15}$ This shows that mass spectrometry imaging methods show promise for integration with routine police recovery procedures.

Another technique that can provide high resolution images of ionised molecules is secondary ion mass spectrometry (ToF-SIMS). Previous work $^{28,29}$ has demonstrated that ToF-SIMS can provide high resolution images of fingerprints and is compatible with various substrates and development reagents. Whilst most MALDI and DESI systems lack the spatial resolution to visualise third level detail (pore structure) within a fingerprint, this has been previously demonstrated with ToF-SIMS. However, a drawback of ToF-SIMS is the limited sensitivity to organic species, due to fragmentation.
Recent advances in the ion sources used in ToF-SIMS systems have led to the development of water cluster SIMS, which offers enhanced sensitivity to intact organic molecules. ${ }^{30,31}$

In this work, we apply MALDI, DESI, ToF-SIMS and water cluster SIMS imaging to fingerprint samples collected from drug users as well as from participants (non-drug users) who were asked to handle cocaine. We show how mass spectrometry imaging (MSI) of fingerprints can help distinguish between administration and contact with cocaine.

\section{Materials and methods}

\section{Materials}

Illicit drug standards (cocaine and benzoylecgonine (BZE)) were obtained from Cerilliant (Dorset, UK) and cocaine hydrochloride powder (99\% purity) was sourced from Sigma-Aldrich (UK). To prepare all solutions and solvent mixtures, Optima ${ }^{\mathrm{TM}}$ LC-MS grade solvents: methanol $(\mathrm{MeOH})$, acetonitrile (ACN), and water $\left(\mathrm{H}_{2} \mathrm{O}\right)$ were used (Fischer Scientific, Leicestershire, $\mathrm{UK})$.

\section{Sample collection}

A favourable ethical opinion for collection and analysis of samples from study participants was granted from the National Research Ethics Service (NRES - REC reference: 14/ LO/0346). Informed consent was obtained from all human subjects. Fingerprint samples were collected on SuperFrost Plus ${ }^{\mathrm{TM}}$ Adhesion slides (Thermo Scientific, Loughborough, UK) from the left thumb and index fingers for DESI-MS and MALDI-MS, respectively, and on a silicon wafer from the middle and ring fingers for ToF-SIMS and water cluster SIMS. Samples were collected from participants attending a drug rehabilitation clinic and who testified taking cocaine within the last 24 hours. Fingerprints were collected "as presented" and "after handwashing". For the "as presented" samples, participants deposited their fingerprints without any wiping or hand washing. For the "after handwashing" samples, participants washed their hands (soap used and duration of handwashing was not controlled), and dried them completely before wearing nitrile gloves for 10 minutes to induce sweating. All samples were collected on each substrate using kitchen scales, pressing down until a reading of 1000 to $1200 \mathrm{~g}$ was achieved and then holding for 10 seconds. Note that the route of cocaine administration, and time of administration was not monitored.

A second set of fingerprint samples was collected from nondrug users who purposely touched cocaine. Two sources of cocaine were used - laboratory grade cocaine hydrochloride powder (described above) and cocaine seized (41\% purity with traces of benzoylecgonine, ecgonine methyl ester, caffeine, lignocaine and levamisole) by the Irish police and stored at Forensic Science Ireland (FSI). In these experiments, participants were asked to touch a pre-weighed mass of cocaine (0.5 $\mathrm{mg}$ or $2 \mathrm{mg}$ ) and spread the powder over all their righthand fingers for approximately 10 seconds. Participants then dusted the excess off to remove large particles, and deposited 
fingerprints using the same procedures described above for both "as presented" and "after handwashing".

\section{Instrumentation}

Desorption electrospray ionisation (DESI). DESI analysis of fingerprint samples was carried out at the National Physical Laboratory (NPL). The DESI 2D ion source (Prosolia) was coupled to a Waters SYNAPT G2-Si High Definition Mass Spectrometer (Waters, UK). The movement of the DESI stage was controlled using the Omnispray software. Data was acquired in positive ion mode using a $5 \mathrm{kV}$ capillary voltage, $50 \mathrm{~V}$ cone voltage and a capillary temperature of $100{ }^{\circ} \mathrm{C}$. Samples were analysed in full scan mode (mass range of $\mathrm{m} / \mathrm{z}$ 100 to 1200 ) and with a calculated mass resolution of 14000 at $\mathrm{m} / \mathrm{z} 304.15$.

The solvent mixture employed used 90:10 (\%v/v) methanol: water (with $0.1 \%$ formic acid), dispensed with a $2 \mu \mathrm{min}^{-1}$ flow rate using a syringe pump. Optimised DESI operating parameters are described in Table 1.

Matrix assisted laser desorption ionisation (MALDI). A Waters prototype UMALDI ion source was coupled to a Waters SYNAPT G2-Si High Definition Mass Spectrometer (Waters, UK) at the National Physical Laboratory (Teddington, UK). Data was acquired in positive ion mode, in the $\mathrm{m} / \mathrm{z}$ range 100-1200 and with a calculated mass resolution of 13000 at $\mathrm{m} / \mathrm{z}$ 304.15. Imaging regions were selected using HDI Imaging (Waters, UK). Table 2 describes the operating parameters used for this work.

The matrix consisted of $37.5 \mathrm{mg} \mathrm{mL} \mathrm{mL}^{-1}$ of DHB (2,5-dihydroxybenzoic acid) prepared in 50:50 (\%v/v) ACN : water and was deposited on the sample using a pneumatic sprayer (TM Sprayer, HTX Imaging, USA). The sprayer was operated at $75{ }^{\circ} \mathrm{C}$, with a gas pressure of $10 \mathrm{psi}$ and gas flow rate of $2 \mathrm{~L}$ $\mathrm{min}^{-1}$. The matrix was sprayed with a $0.08 \mathrm{~mL} \mathrm{~min}^{-1}$ flow rate, velocity of $1200 \mathrm{~mm} \mathrm{~min}^{-1}$, nozzle height of $40 \mathrm{~mm}$ and 16 passes over the sample.

Time of flight secondary ion mass spectrometry (ToF-SIMS). ToF-SIMS analyses of fingerprints were carried out using an ION-TOF GmbH (Munster, Germany) TOF.SIMS.5 instrument. A $25 \mathrm{keV} \mathrm{Bi}_{3}{ }^{+}$(extracted at $10 \mathrm{kV}$ ) primary ion beam was operated delivering $1.0 \mu \mathrm{A}$ of current with the high current bunched mode. The beam can be scanned over a $100 \times 100 \mu \mathrm{m}$ area but for large area imaging, the MacroRaster mode was employed, where the stage moves under the beam to provide a mosaic of the total area analysed. Images were acquired with 1 scan, 1 shot per pixel, 10 frames per patch, a maximum patch

Table 1 Optimised DESI operating parameters for fingerprint analysis

\begin{tabular}{ll}
\hline Parameter & Setting \\
\hline Distance between spray tip and surface & $2 \mathrm{~mm}$ \\
Impact angle & $65^{\circ}$ \\
Distance between sprayer and MS capillary inlet & $5 \mathrm{~mm}$ \\
Pressure of $\mathrm{N}_{2}$ gas & $80 \mathrm{psi}$ \\
Scan speed & $0.985 \mathrm{~s}$ per scan \\
Pixel size & $100 \mu \mathrm{m} \times 100 \mu \mathrm{m}$
\end{tabular}

Table 2 MALDI operating parameters for fingerprint analysis

\begin{tabular}{ll}
\hline Parameter & Setting \\
\hline Laser energy & 350 (arbitrary units) \\
Laser repetition rate & $2500 \mathrm{~Hz}$ \\
Scan time & $0.1 \mathrm{~s}$ \\
UV laser & $355 \mathrm{~nm}$ \\
Pixel size & $50 \mu \mathrm{m} \times 50 \mu \mathrm{m}$ \\
Raster speed & $0.5 \mathrm{~mm} \mathrm{~s}^{-1}$ \\
Scan rate & $10 \mathrm{scans}^{\mathrm{s}} \mathrm{s}$
\end{tabular}

side length of $0.3 \mathrm{~mm}$ and a pixel density of 300 pixel per $\mathrm{mm}$. Images were acquired over a $10 \times 10 \mathrm{~mm}$ area. Data was acquired in positive mode and with a $100 \mu$ s TOF cycle. All spectra and ion images were analysed and generated using "SurfaceLab 6.5" provided by ION-TOF.

Water cluster SIMS. Water cluster SIMS was performed on a J105 SIMS (Ionoptika Ltd), as described previously. ${ }^{32}$ The primary ion beam used was a $70 \mathrm{kV}$ gas cluster ion beam fitted with a water source. ${ }^{30,33}$ The water source replaces a standard gas cluster ion beam (GCIB) source to enable both wet and dry gas sources to be used. A $70 \mathrm{kV}\left(\mathrm{H}_{2} \mathrm{O}\right)_{29000}{ }^{+}$beam was used for this study with a current of $14 \mathrm{pA}$, focused to a spot size of $10 \mu \mathrm{m}$ with a duty cycle of $50 \%$.

SIMS spectra were acquired in positive ion mode over an area of $18 \times 6 \mathrm{~mm}^{2}$, covering approximately half of the fingerprint. Each $500 \times 500 \mu \mathrm{m}$ tile consisted of $32 \times 32$ pixels at 400 shots per pixel, giving a pixel size of $15.6 \mu \mathrm{m}$ and a total ion dose of $7.2 \times 10^{11}$ ions per $\mathrm{cm}^{2}$. A sample bias of $+10 \mathrm{~V}$ was used to promote ion extraction. No other charge neutralisation was used.

The mass range of the acquired spectra was $m / z 50-900$, and the mass resolution was $m / \Delta m=10088$, as measured on the $m / z 304.15$ signal.

\section{Data processing}

Waters .raw files required conversion into appropriate .imzML files for data analysis. This required a two-step conversion where raw data files were converted to .mzml, then to .imzml files using MS convert software $^{34}$ and imzml converter, ${ }^{35}$ respectively. Finally, data was manipulated and analysed using SpectralAnalysis version $1.2^{36}$ through MATLAB. Prior to generating a mean spectrum, an interpolation rebin method was applied with a bin size of 0.001 . Extracted ion images were produced from the mean spectra.

The calculated monoisotopic masses for the analytes detected across all techniques are shown in ESI Table $1 . \dagger$

\section{Results}

\section{'As presented' (no handwashing)}

DESI images of a fingerprint sample taken from a drug user who testified taking cocaine in the last 24 hours are shown in Fig. 1. Images corresponding to (A) $m / z 494.566$ (assigned to a ditallowdimethylammonium (DTDMAC) ion as $[\mathrm{M}]^{+}$), (B) $\mathrm{m} / \mathrm{z}$ 

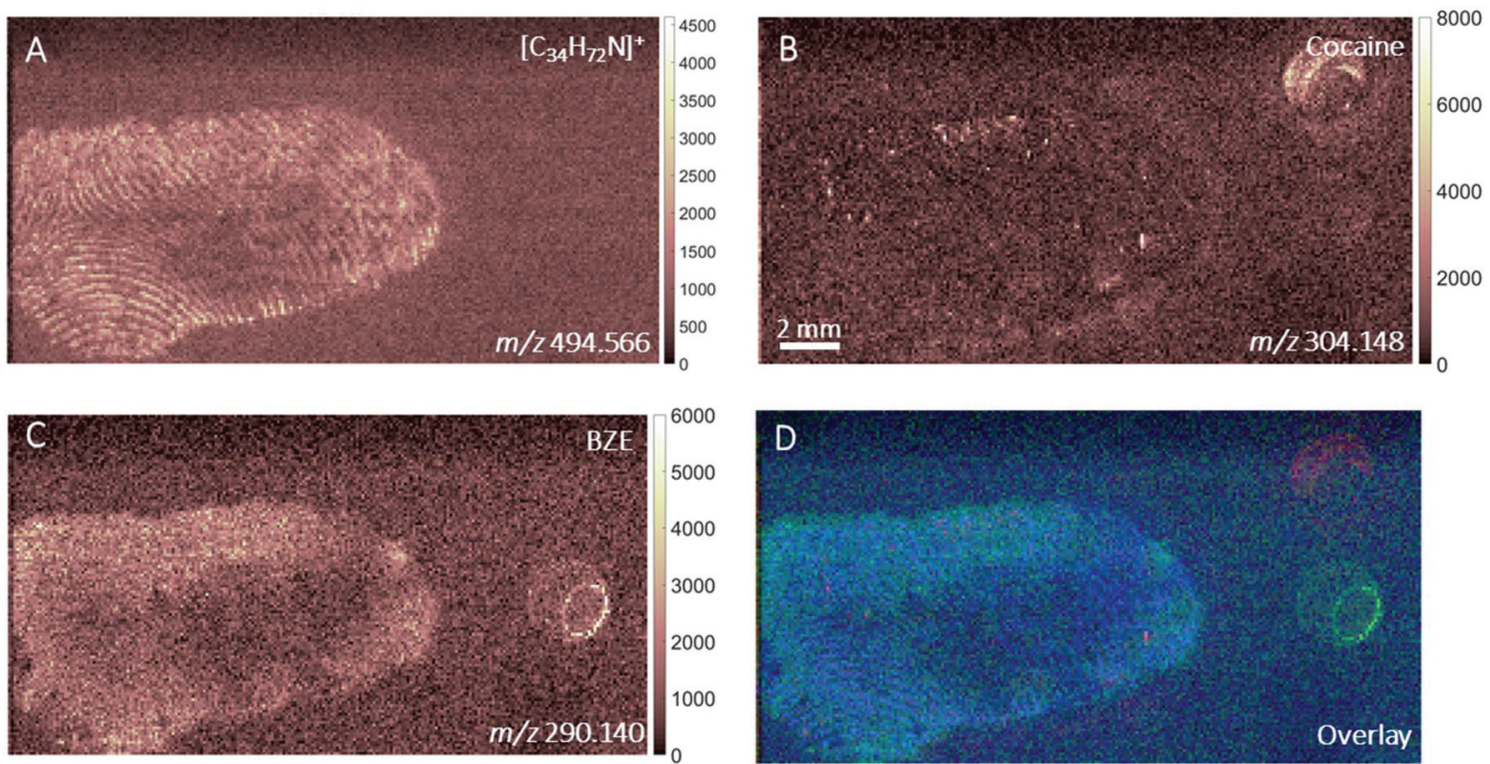

Fig. 1 Ion images obtained with DESI-MS of (A) ditallowdimethylammonium ion $(\mathrm{m} / \mathrm{z} 494.566, \Delta m=0.16 \mathrm{ppm}),(\mathrm{B}) \mathrm{cocaine}(\mathrm{m} / \mathrm{z} 304.148, \Delta m=$ $20 \mathrm{ppm}$ ) and (C) BZE ( $\mathrm{m} / \mathrm{z} 290.140, \Delta m=5 \mathrm{ppm})$ and (D) overlay of cocaine (red), BZE (green) and ditallowdimethylammonium ion (blue) taken from an individual who ingested cocaine, pre-hand washing ("as presented").

304.148 (assigned to cocaine as $\left.[\mathrm{M}+\mathrm{H}]^{+}\right)$, (C) $\mathrm{m} / \mathrm{z} 290.140$ (assigned to BZE as $[\mathrm{M}+\mathrm{H}]^{+}$, a metabolite of cocaine) and (D) overlay are presented. Note that the circles on the right-hand side of the ion images relate to spotted standards of $50 \mathrm{pg}$ (5 $\mathrm{ng} \mathrm{m} \mathrm{m}^{-1}$ ) cocaine (top right), BZE (middle right), and ecgonine methyl ester (bottom right; not used for these analyses) added to confirm peak assignment. The ion image generated from the peak closest to the monoisotopic mass of cocaine (theoretical mass $m / z$ 304.1543) does not provide a clear fingerprint image due to low abundance (Fig. 1B). Fig. 1C shows a uniform distribution of BZE signal over the fingerprint area. Fig. 1D shows an overlay of the cocaine, BZE and DTDMAC images. Although the spatial resolution is insufficient to clearly reveal fingerprint ridges, the intensity distribution of
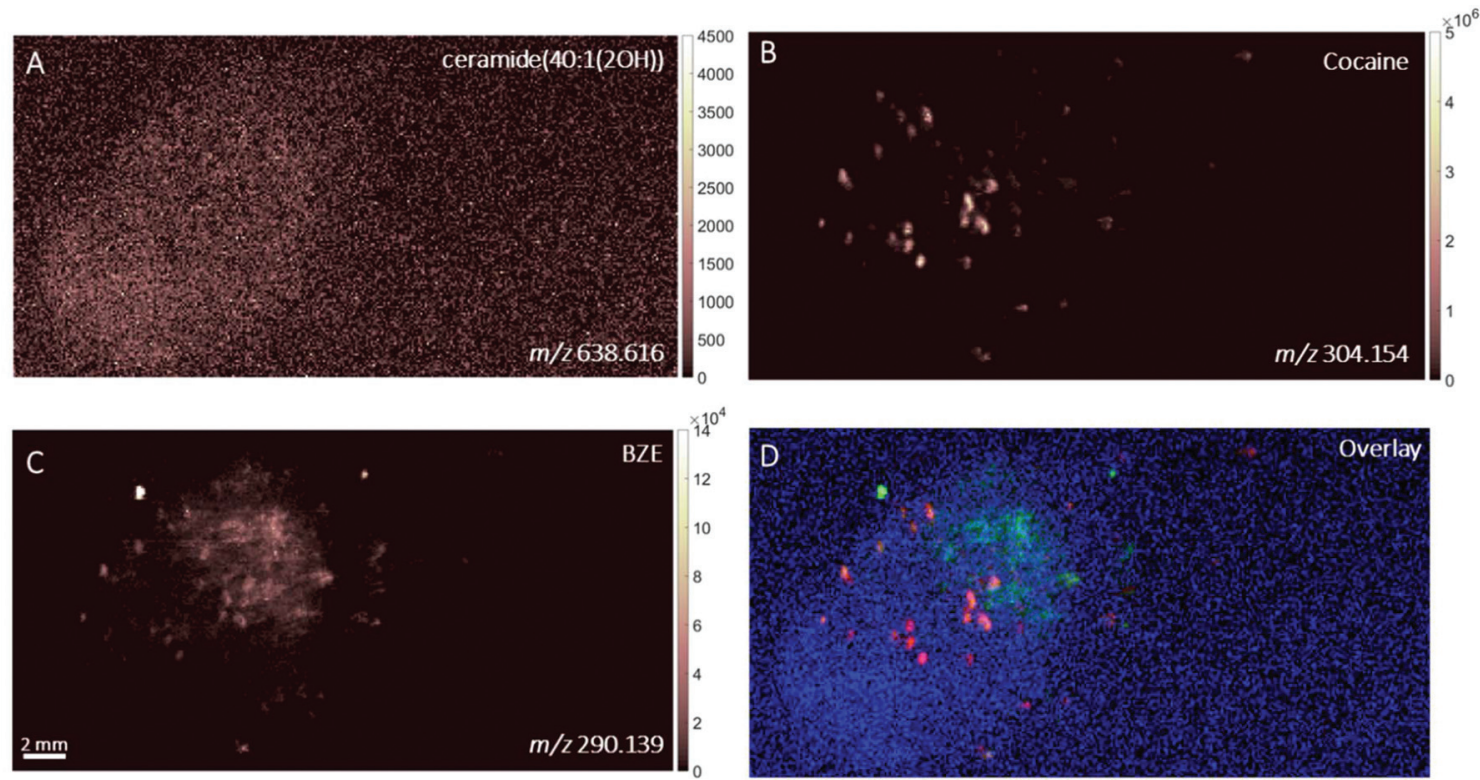

Fig. 2 Ion images obtained with DESI of (A) ceramide $(40: 1(2 \mathrm{OH}))(\mathrm{m} / \mathrm{z} 638.616, \Delta m=12 \mathrm{ppm})$, (B) cocaine signal $(\mathrm{m} / \mathrm{z} 304.154, \Delta m=0 \mathrm{ppm}),(\mathrm{C})$ BZE signal ( $m / z$ 290.139, $\Delta m=1 \mathrm{ppm}$ ) and (D) overlay of the ion images of cocaine (red), BZE (green) and ceramide (blue) taken from an individual after contact with seized cocaine, pre-hand washing ("as presented"). 
BZE across the area of the fingerprint correlates well with the DTDMAC image. This is consistent with BZE being excreted in eccrine sweat after cocaine use.

Fig. 2 shows the ion images generated using DESI from a fingerprint sample collected "as presented" from a participant who was asked to handle $2 \mathrm{mg}$ of seized cocaine. The ceramide signal $(\mathrm{m} / \mathrm{z}$ 638.616) does not show defined ridges, perhaps due to low abundance in the fingerprint or ion suppression. This was also observed for other endogenous compounds expected to be present in fingerprint secretions, based on previous work. ${ }^{16}$ The detection of BZE is consistent with the pre- vious observation that despite a lack of cocaine administration, it is possible to see this metabolite of cocaine after contact with the illicit drug. ${ }^{5}$ Unlike the fingerprint from the drug user, the cocaine image is more intense than that of BZE (note the different intensity scales). Additionally, these images show that presence of cocaine and BZE in fingerprints after dermal contact can be observed as discrete hot spots. This contrasts with the continuous distribution of BZE correlating with the fingerprint matrix observed in Fig. 1.

Fig. 3 shows the ion images from a fingerprint collected as presented from a cocaine user who testified taking the sub-

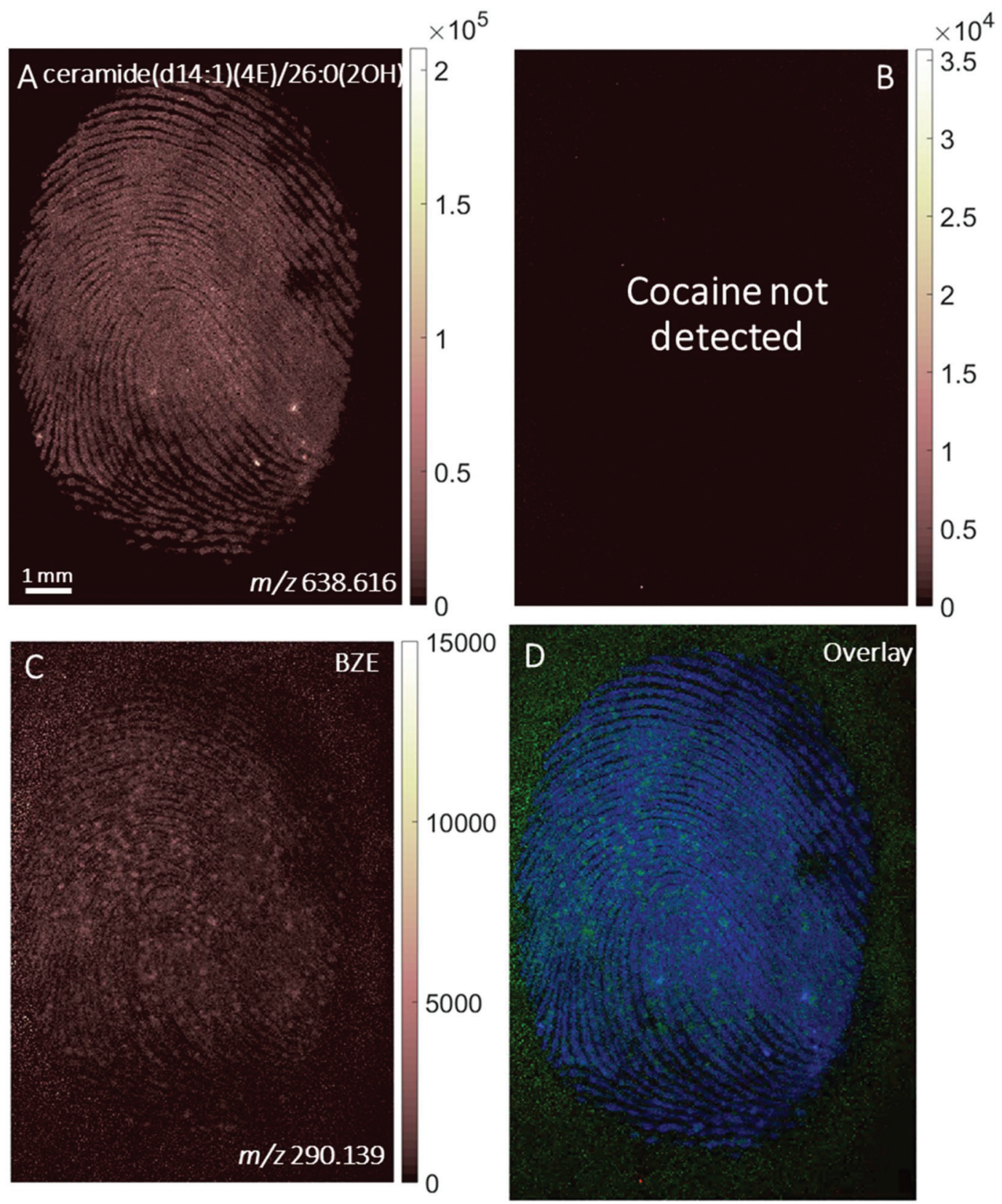

Fig. 3 Ion images obtained with MALDI of (A) ceramide $(40: 1(2 \mathrm{OH}))(\mathrm{m} / \mathrm{z} 638.616, \Delta m=12 \mathrm{ppm})$, (B) $\mathrm{m} / \mathrm{z} 304.154$ (expected $\mathrm{m} / \mathrm{z}$ of cocaine ion), (C) BZE ( $m / z$ 290.139, $\Delta m=1 \mathrm{ppm}$ ) and (D) overlay of cocaine (red), BZE (green) and ceramide (blue) taken from an individual who ingested cocaine, pre-hand washing ("as presented"). 
stance in the last 24 hours and analysed with MALDI. No signals at $\mathrm{m} / \mathrm{z} 304.154$ (corresponding to cocaine) were found in this sample, but BZE was present. This is consistent with the observations made above for DESI, where the BZE ion image is more intense than the cocaine ion image following ingestion of cocaine. The resolution of the image is sufficient to show that the BZE co-locates with the fingerprint ridges, as shown in Fig. 3D.

MALDI images of a fingerprint sample collected from a participant who touched $0.5 \mathrm{mg}$ cocaine hydrochloride powder is presented in Fig. 4. Paper spray analysis of the cocaine powder used in this experiment showed the presence of BZE, explaining the presence of this metabolite in this fingerprint sample. ${ }^{5}$ This is further supported by the co-localisation of the cocaine and BZE signals shown in Fig. 4C and D. After contact with cocaine, the signals corresponding to cocaine and BZE do not correlate spatially with the ceramide image and are observed as discrete hot spots, as clearly demonstrated in Fig. 4D. The intensity of the cocaine image is brighter than that of BZE, corroborating the DESI measurements presented in Fig. 2.

To gain a high-resolution view of these samples, ToF-SIMS was employed to visualise the relationship between the drug analytes and the pore structure of the fingerprint, as shown in Fig. 5. Here, a fingerprint collected from a non-drug user, as presented, after contact with $2 \mathrm{mg}$ of cocaine hydrochloride powder is shown. The ion image for the sum of ditallowdimethylammonium ions $(\mathrm{m} / \mathrm{z} 494.52,522.55,550.58)$, previously identified in fingerprint samples, ${ }^{16,37}$ is shown in Fig. $5 \mathrm{C}$ and
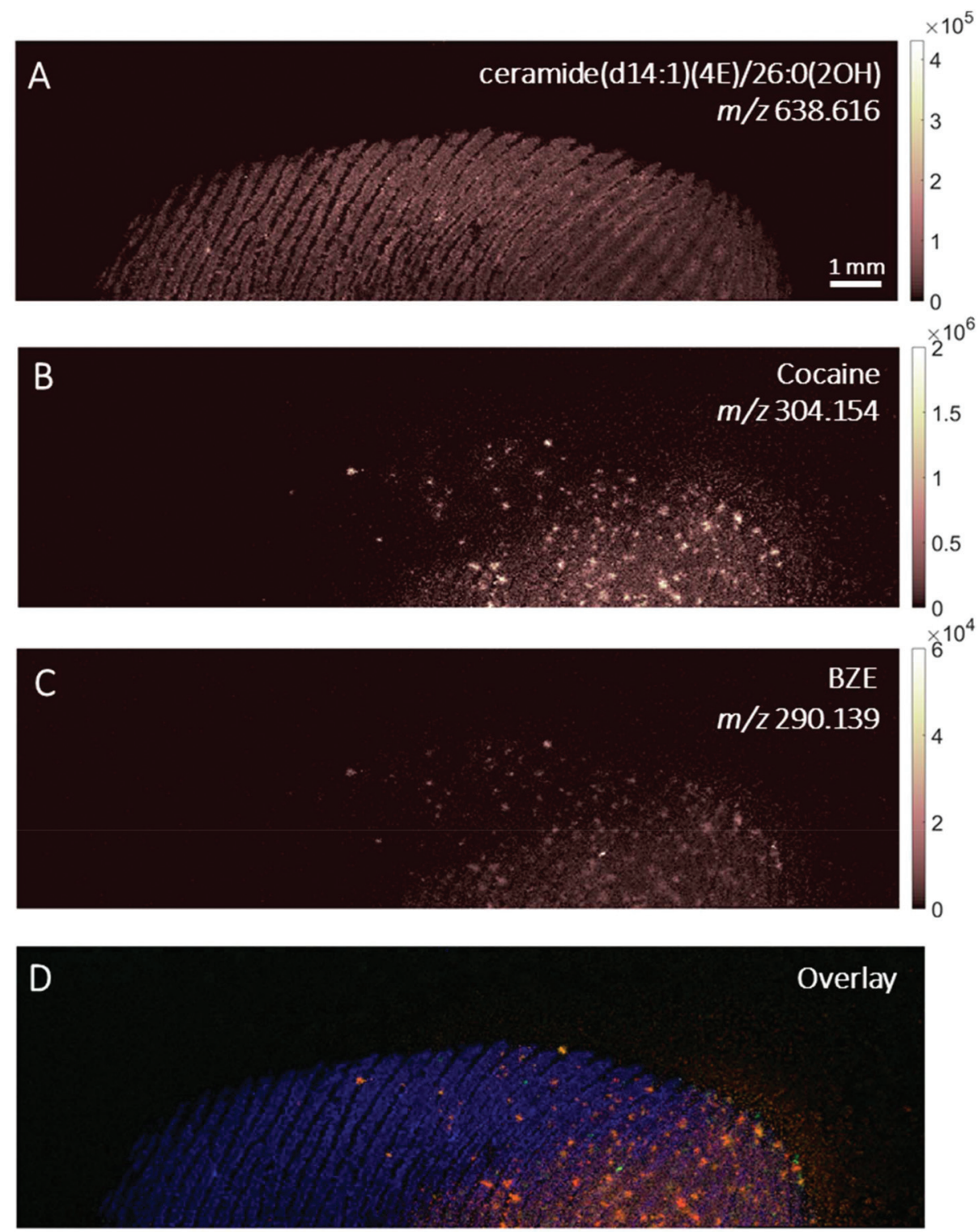

Fig. 4 Ion images obtained with MALDI of (A) ceramide(40:1(2OH)) $(\mathrm{m} / \mathrm{z} 638.616, \Delta m=12 \mathrm{ppm})$, (B) cocaine $(\mathrm{m} / \mathrm{z} 304.154, \Delta m=3 \mathrm{ppm})$ and $(\mathrm{C})$ BZE $(\mathrm{m} / \mathrm{z} 290.139, \Delta m=1 \mathrm{ppm})$ and (D) overlay of cocaine (red), BZE (green) and ceramide (blue) from a fingerprint sample collected from an individual after contact with $0.5 \mathrm{mg}$ of cocaine power, pre-handwashing ("as presented"). 

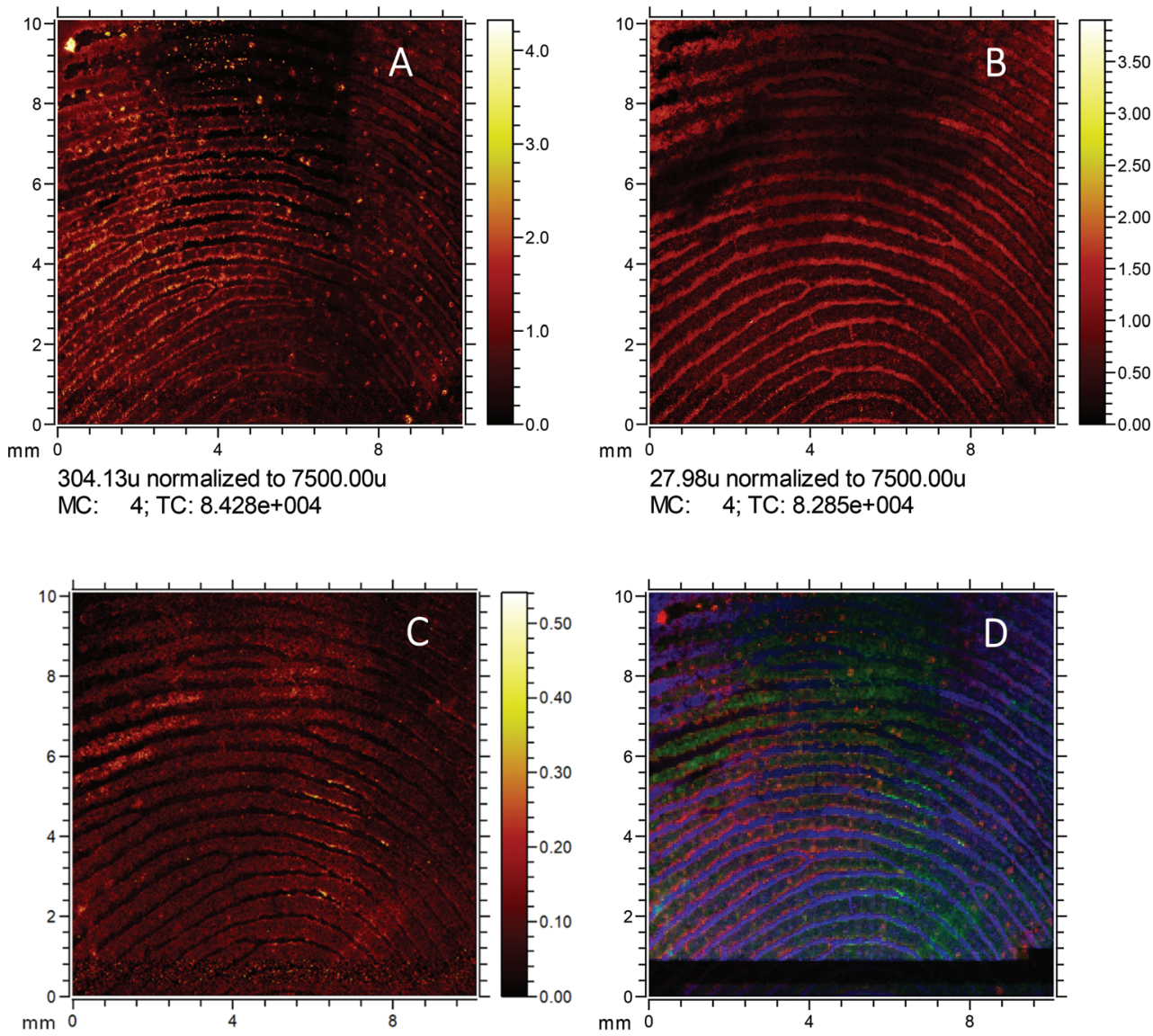

Sum of: $494.52 \mathrm{u}$ normalized to $7500.00 \mathrm{u}, 522.55 \mathrm{u}$ normalized to $7500.00 \mathrm{u}, 550.58 \mathrm{u}$ normalized to $7500.00 \mathrm{u}$

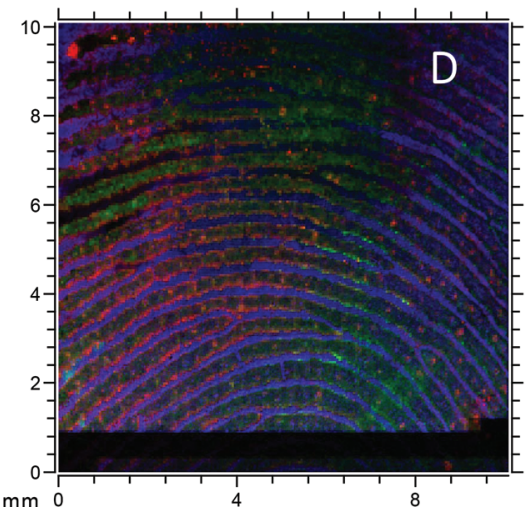

Overlay of $304.13 u$, Sum of: $494.52 u, 522.55 u$, 550.58u, 27.98u

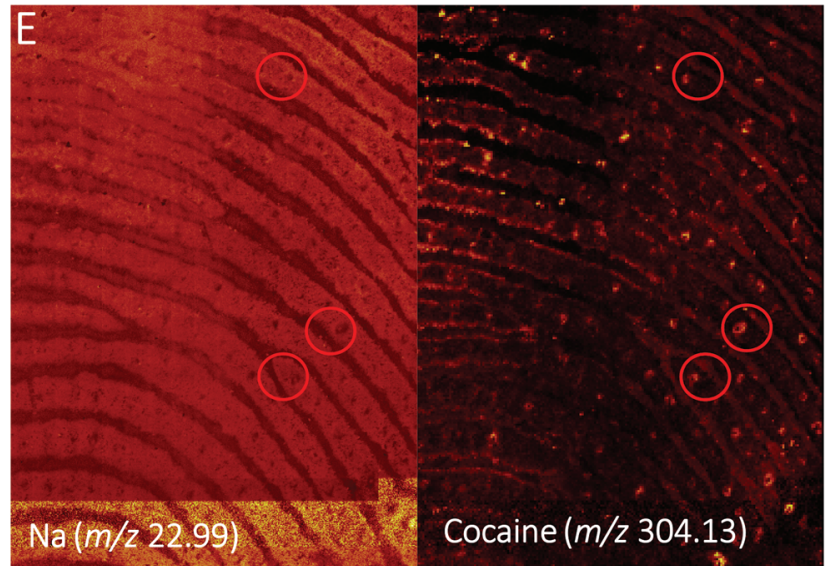

Fig. 5 Ion images obtained with ToF-SIMS (normalised to the total ion count) of (A) cocaine $(\mathrm{m} / z$ 304.13, $\Delta m=79 \mathrm{ppm})$, (B) Si ${ }^{+}$signal from substrate $(m / z 27.98, \Delta m=110 \mathrm{ppm})$, (C) sum of ditallowdimethylammonium ions $(\mathrm{m} / \mathrm{z} 494.52,522.55,550.58, \Delta \mathrm{m}=93 \mathrm{ppm}, 90 \mathrm{ppm}$ and $88 \mathrm{ppm}$, respectively), (D) overlay of images $A($ red), $C$ (green) and B (blue) and (E) close-up of sodium ion image $(\mathrm{m} / \mathrm{z} 22.99, \Delta \mathrm{m}=13 \mathrm{ppm})$ and cocaine $(\mathrm{m} / \mathrm{z}$ 304.13) showing the pore structure of an individual who was in contact with $2 \mathrm{mg}$ of cocaine hydrochloride powder.

shows the location of the fingerprint ridges, which are anticorrelated with the Si distribution (from the substrate). The image of $\mathrm{m} / \mathrm{z} 304.13$ (assigned to cocaine $[\mathrm{M}+\mathrm{H}]^{+}$) is overlaid with the ditallowdimethylammonium ions and $\mathrm{Si}^{+}$substrate signal in Fig. 5D. BZE is also detected (not shown), but at a lower signal intensity when compared to cocaine, as observed using both MALDI and DESI. The ion image shown in Fig. 5A shows that the cocaine is not uniformly distributed within the 
ridges, and towards the right-hand side and lower part of this image, this analyte is residing in the valleys as well as on the ridges. Fig. $5 \mathrm{E}$ shows the distribution of $\mathrm{Na}^{+}$for a selected area of the fingerprint, which clearly shows the pore structure. On the right of Fig. 5E, the cocaine signal in this region is also presented, showing that the cocaine co-locates with the pores of the fingerprint.

A fingerprint collected from a drug user was also imaged using ToF-SIMS. BZE was detected via the protonated molecular ion $(\Delta m=99 \mathrm{ppm})$ and main fragment ion, but as reported previously, ${ }^{17}$ the technique lacked sensitivity to provide satisfactory images of either the cocaine or BZE distribution (see ESI Fig. $1 \dagger$ ).

One of the limitations of conventional ToF-SIMS primary beam analysis is low ion yield from organic molecules due to high levels of fragmentation. Advances in ion gun technology has recently resulted in the introduction of water cluster guns, which have shown promising results for analysis of organic and biological molecules. ${ }^{30,38}$ Here we demonstrate the first application of a water cluster SIMS source to a fingerprint sample. Fig. 6 shows the ion images of (A) $\mathrm{m} / \mathrm{z} 304.15$ (assigned to cocaine as $[\mathrm{M}+\mathrm{H}]^{+}$), (B) $m / z 290.14$ (assigned to BZE as $\left.[\mathrm{M}+\mathrm{H}]^{+}\right)$, (C) an ion assigned as ditallowdimethylammonium $(\mathrm{m} / \mathrm{z} 494.60)$ seen previously in fingerprints ${ }^{16,37}$ and (D and E) close-up of Fig. 6B (BZE) and Fig. 6C (fingerprint ridges) from a fingerprint sample collected from a cocaine user, as presented.

Using $\mathrm{a} \mathrm{Bi}_{3}{ }^{+}$ion gun, neither cocaine or $\mathrm{BZE}$ could be imaged in fingerprint samples collected under the same conditions. However, using the water cluster primary ion beam,
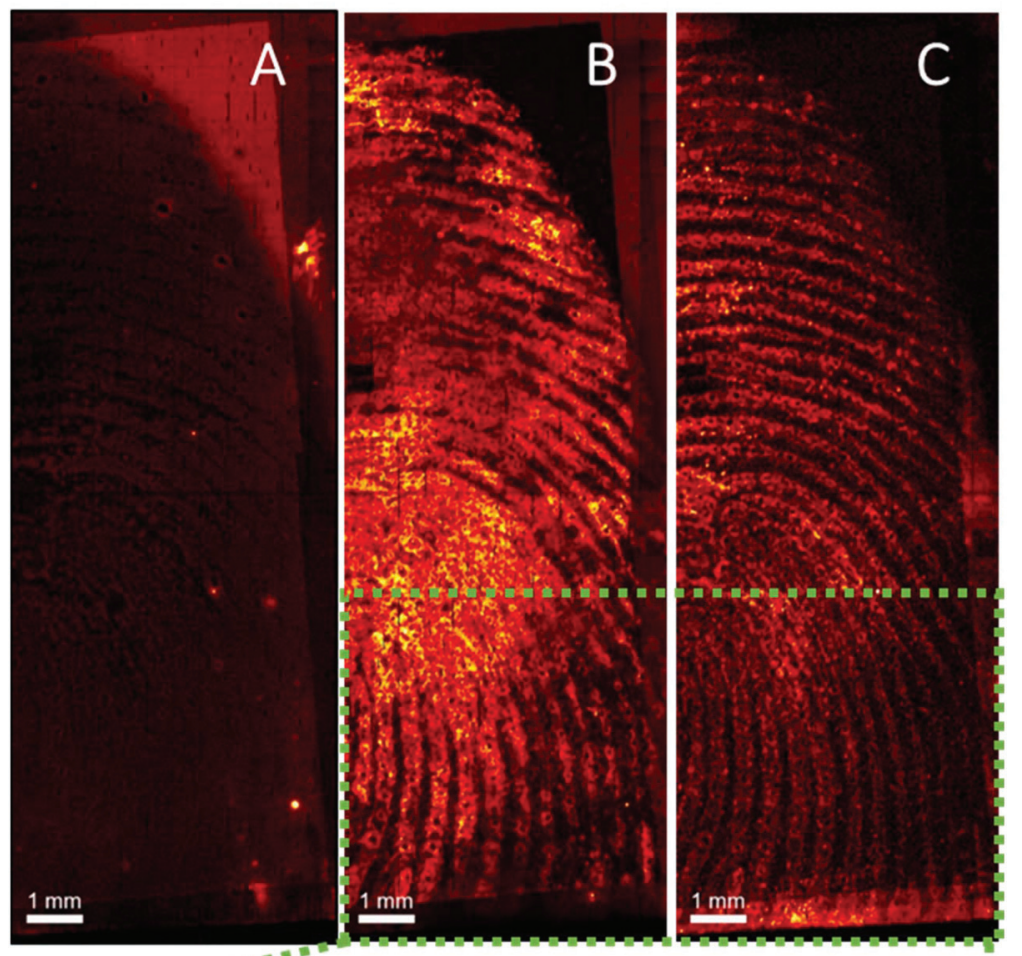
BZE was detected and found to correlate with the fingerprint ridges as presented in Fig. 6, highlighting the enhanced sensitivity provided by the water cluster beam. As observed with DESI and MALDI, fingerprints collected from cocaine users as presented, show a high BZE-to-cocaine image intensity ratio over the area of the fingerprint. The ridge details are clearly observable, and one can even distinguish sweat pores in some areas of the fingerprint (Fig. 6D and E).

\section{'After handwashing'}

The images provided by DESI, MALDI and SIMS so far show that mass spectrometry images of fingerprints can be used to distinguish between contact and ingestion of an illicit drug based on the relative intensity of cocaine and BZE images and the distribution of these analytes over the fingerprint area. To test whether this observation holds also for fingerprints donated after handwashing, a second set of fingerprint samples were collected after handwashing from both cocaine users and participants who were asked to handle cocaine and analysed using MALDI. The images for cocaine and BZE and corresponding overlays are shown in Fig. 7 (administration) and Fig. 8 (contact).

In both these samples, the findings are consistent with previous observations made for 'as presented' samples where cocaine provides the highest ion intensity for the dermal contact scenario, whilst BZE provides the highest ion intensity in the area of the fingerprint deposition when cocaine was administered. Fig. 9 compares the overlays of cocaine and BZE with ceramide in each of the two scenarios being studied here. One interesting observation is that for dermal contact (Fig. 9A) cocaine can be found both on and off the fingerprint ridges, even after handwashing. Additionally, cocaine does not show even coverage of the entire fingerprint area. Conversely, BZE excreted after cocaine administration (Fig. 9B) is co-located with the ridges and is more uniformly distributed across the fingerprint.

\section{Discussion}

We propose that the spatial distribution of cocaine and BZE in a fingerprint can be used to discriminate between cocaine administration ( $n=4$ participants, 1 fingerprint per participant) and dermal contact ( $n=4$ participants, 1 fingerprint per participant). The results above demonstrate that despite the different imaging scales, modes of ionisation and detection capabilities of the MSI techniques employed here, MALDI, DESI and SIMS were all capable of detecting differences in fingerprints deposited under these circumstances. ESI Tables 2 and $3 \dagger$ show a comparison of the resulting ion images for all
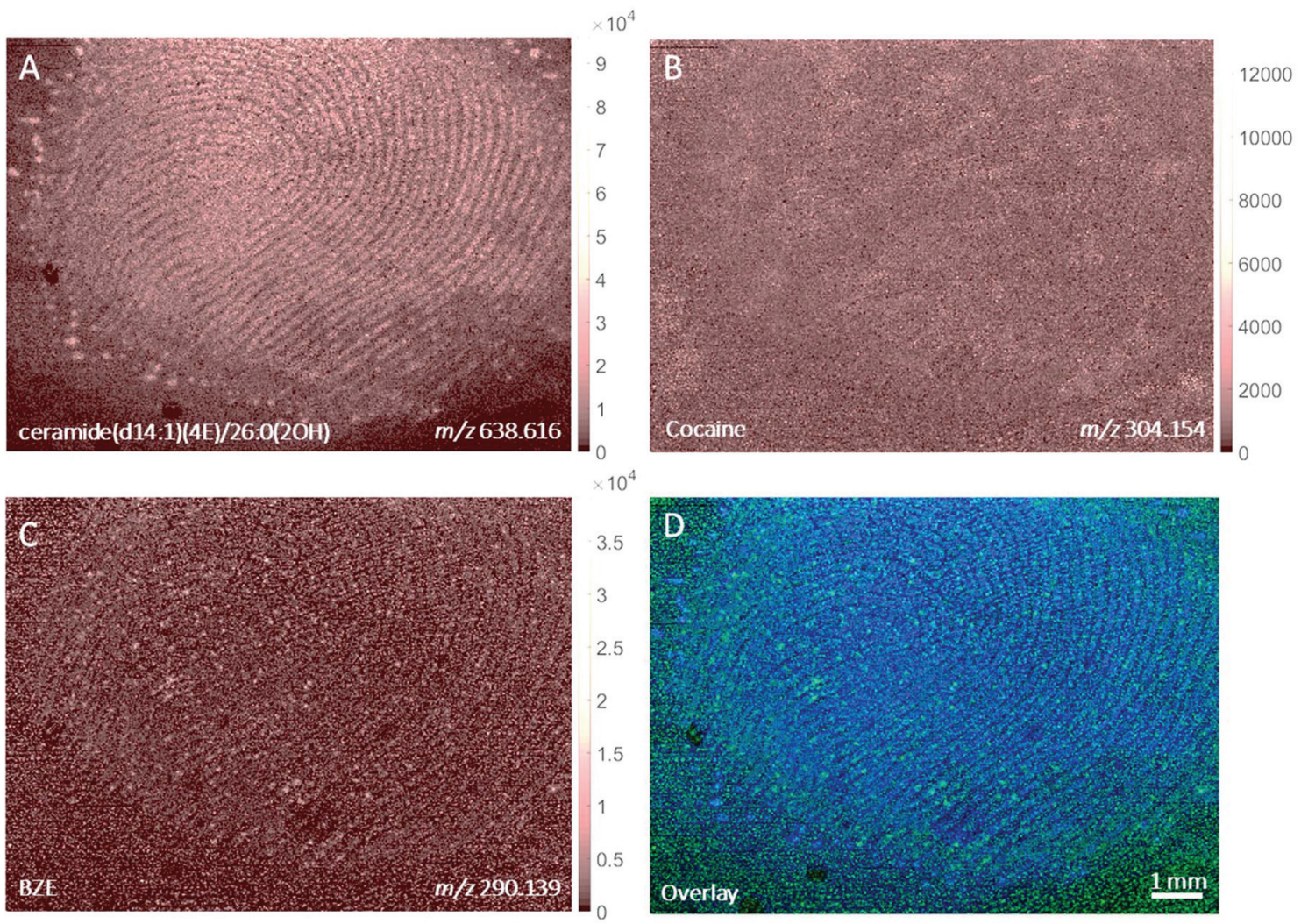

Fig. 7 Ion images obtained with MALDI of (A) ceramide(40:1(2OH)) $(\mathrm{m} / \mathrm{z} 638.616, \Delta m=12 \mathrm{ppm})$, (B) cocaine $(\mathrm{m} / \mathrm{z} 304.154, \Delta m=0 \mathrm{ppm})$ and $(\mathrm{C})$ BZE $(m / z$ 290.139, $\Delta m=1 \mathrm{ppm})$ and (D) overlay of BZE (green) and ceramide (blue) from a fingerprint sample collected after handwashing from a participant who had administered cocaine. 

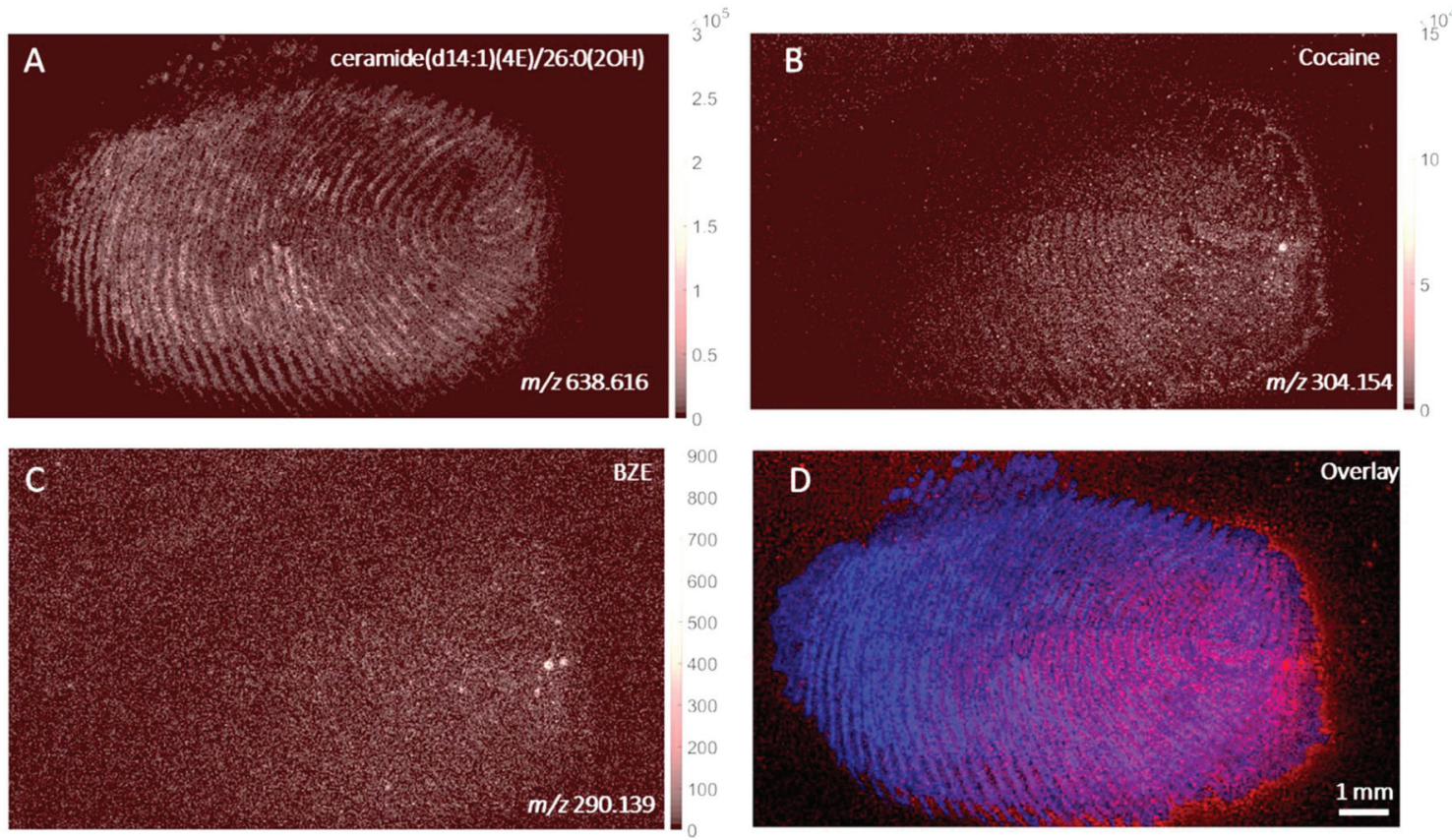

Fig. 8 Ion images obtained with MALDI of (A) ceramide(40:1(2OH)) $(\mathrm{m} / \mathrm{z} 638.616, \Delta m=12 \mathrm{ppm}),(\mathrm{B})$ cocaine $(\mathrm{m} / \mathrm{z} 304.154, \Delta m=0 \mathrm{ppm})$ and $(\mathrm{C})$ BZE $(m / z 290.139, \Delta m=1 \mathrm{ppm})$ and (D) overlay of cocaine (red) and ceramide (blue) from a fingerprint sample collected after handwashing from a participant who had touched $0.5 \mathrm{mg}$ of cocaine powder.

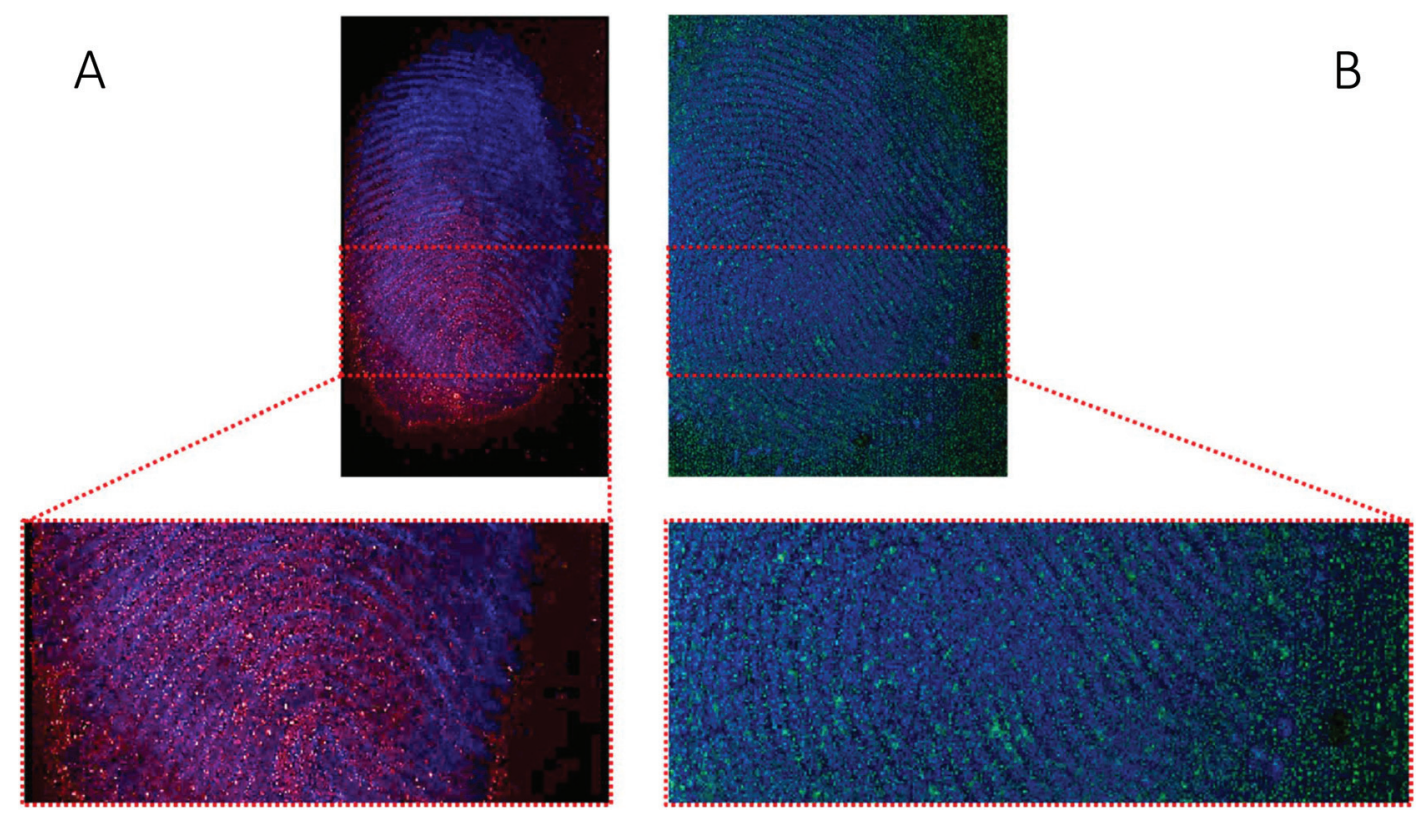

Fig. 9 Ion images obtained with MALDI of (A) fingerprint sample collected after handwashing from a participant who had touched 0.5 mg of cocaine powder (Fig. 8, overlay of cocaine (red) and ceramide (blue)) and (B) fingerprint sample collected after handwashing from a cocaine user (Fig. 7, overlay of BZE (green) and ceramide (blue)).

scenarios and techniques to aid this discussion. Ingestion can be characterised by a higher intensity of BZE (relative to cocaine) in the area of fingerprint deposition, as well as an even distribution of these analytes across the fingerprint and on the ridges. Contact is characterised by a higher intensity of cocaine (relative to BZE) in the area of fingerprint deposition, as well as analyte distribution that does not directly correlate with the flow of fingerprint ridges. Therefore if a fingerprint is deposited under controlled circumstances (e.g. at a crime scene), it may be possible to deduce whether or was deposited 
after a donor had used or consumed cocaine. For operational use, future work would be required to establish whether analytes are delocalised after the fingerprint is developed by routine forensic procedures.

Imaging of samples provided after handwashing suggest that the differences between contact and ingestion still hold even if hands are washed. It is therefore worth exploring whether imaging mass spectrometry could be used as a confirmation test in drug testing, in cases where there is doubt over the result.

To probe the general applicability of this approach, each technique has imaged one fingerprint from four different drug users. It is well known that fingerprint sampling is difficult to standardise. ${ }^{39,40}$ Previous work using traditional bulk analyses have shown that the mass of illicit drug or metabolite detected in a fingerprint even from the same drug user can be highly variable, making it difficult to discriminate between those who have ingested cocaine and those who have been in contact with the illicit drug. ${ }^{1,2}$ This study therefore makes no attempt to compare the sensitivity of MSI imaging techniques for this application.

SIMS, MALDI and DESI have been demonstrated previously for imaging fingerprints, but their use in distinguishing fingerprints from contact and ingestion scenarios is novel. The use of a water cluster ion gun for fingerprint imaging is also novel, and enables not only the detection of BZE in samples collected as presented from a cocaine user, but also spatial resolution sufficient to see the pore structure of the fingerprint.

One final observation is the ability to distinguish cocaine contact from cocaine ingestion by observing the relative intensity of cocaine and BZE ion images across the entire fingerprint. Using the data from Jang et al., ${ }^{5}$ which uses bulk analysis, unless hands are washed, it is not possible to observe a difference between contact and ingestion. This dataset implies that regardless of whether the fingers are washed prior to fingerprint donation, analytes can form hotspots on the fingerprint after contact with cocaine. Techniques such as paper spray or liquid chromatography-mass spectrometry (LC-MS), which have been explored for illicit drug detection in fingerprints, rely on bulk analysis and would therefore be oblivious to these hotspots. Thus, quantitative measurements and ratio of parent drug to metabolite may not be representative. In contrast, MSI techniques can separate hotspots from the main fingerprint matrix. We propose that this is a suitable strategy for distinguishing between a fingerprint produced by contact, and a fingerprint produced by ingestion.

\section{Conclusions}

We propose that the spatial distribution of cocaine and its metabolite, BZE in a fingerprint can be used to discriminate between administration and dermal contact. Ingestion can be characterised by a higher intensity of BZE (relative to cocaine) in the fingerprint ridges, as well as an even distribution of these analytes across the fingerprint deposition. Contact is characterised by a higher intensity of cocaine (relative to BZE) in the fingerprint ridges, as well as analyte distribution that does not directly correlate with the flow of fingerprint ridges. Therefore it may be possible to deduce whether or was deposited after a donor had used or consumed cocaine, even if the fingerprint is deposited under uncontrolled conditions.

\section{Conflicts of interest}

There are no conflicts to declare.

\section{Acknowledgements}

The authors would like to acknowledge Brian Gibson and Sheila Willis from Forensic Science Ireland for giving access to street purity cocaine.

\section{References}

1 C. Costa, et al., Rapid, Secure Drug Testing Using Fingerprint Development and Paper Spray Mass Spectrometry, Clin. Chem., 2016, 63(11), 1745-1752.

2 M. Ismail, et al., Noninvasive Detection of Cocaine and Heroin Use with Single Fingerprints: Determination of an Environmental Cutoff, Clin. Chem., 2018, 64(6), 909-917.

3 J. Czerwinska, et al., Detection of mephedrone and its metabolites in fingerprints from a controlled human administration study by liquid chromatography-tandem mass spectrometry and paper spray-mass spectrometry, Analyst, 2020, 145(8), 3038-3048.

4 C. Costa, et al., Paper spray screening and liquid chromatography/mass spectrometry confirmation for medication adherence testing: A two-step process, Rapid Commun. Mass Spectrom., 2021, 35(S2), e8553.

5 M. Jang, et al., On the relevance of cocaine detection in a fingerprint, Sci. Rep., 2020, 10(1), 1974.

6 C. Huynh, et al., Forensic Identification of Gender from Fingerprints, Anal. Chem., 2015, 87(22), 11531-11536.

7 L. S. Ferguson, et al., Direct detection of peptides and small proteins in fingermarks and determination of sex by MALDI mass spectrometry profiling, Analyst, 2012, 137(20), 4686-4692.

8 Z. Zhou and R. N. Zare, Personal Information from Latent Fingerprints Using Desorption Electrospray Ionization Mass Spectrometry and Machine Learning, Anal. Chem., 2017, 89(2), 1369-1372.

9 C. Heaton, et al., Investigating sex determination through MALDI MS analysis of peptides and proteins in natural fingermarks through comprehensive statistical modelling, Forensic Chem., 2020, 20, 100271.

10 W. van Helmond, et al., Chemical profiling of fingerprints using mass spectrometry, Forensic Chem., 2019, 16, 100183. 
$11 \mathrm{~K}$. Asano, et al., Chemical Composition of Fingerprints for Gender Determination, J. Forensic Sci., 2002, 47(4), 1-3.

$12 \mathrm{M}$. de Puit, M. Ismail and X. Xu, LCMS Analysis of Fingerprints, the Amino Acid Profile of 20 Donors, J. Forensic Sci., 2014, 59(2), 364-370.

13 A. de Ronde, et al., The evaluation of fingermarks given activity level propositions, Forensic Sci. Int., 2019, 302, 109904.

14 L. Deininger, et al., Investigation of infinite focus microscopy for the determination of the association of blood with fingermarks, Sci. Justice, 2018, 58(6), 397-404.

15 R. Bradshaw, N. Denison and S. Francese, Implementation of MALDI MS profiling and imaging methods for the analysis of real crime scene fingermarks, Analyst, 2017, 142(9), 1581-1590.

16 M. Bailey, et al., Analysis of Urine, Oral fluid and Fingerprints by Liquid Extraction Surface Analysis Coupled to High Resolution MS and MS/MS - Opportunities for Forensic and Biomedical Science, Anal. Methods, 2016, 8(16), 3373-3382.

17 M. J. Bailey, et al., Rapid detection of cocaine, benzoylecgonine and methylecgonine in fingerprints using surface mass spectrometry, Analyst, 2015, 140(18), 6254-6259.

18 P. Hazarika, et al., Imaging of Latent Fingerprints through the Detection of Drugs and Metabolites, Angew. Chem., Int. Ed., 2008, 47(52), 10167-10170.

19 K. Kuwayama, et al., Effectiveness of saliva and fingerprints as alternative specimens to urine and blood in forensic drug testing, Drug Test. Anal., 2016, 8(7), 644-651.

20 K. Kuwayama, et al., Time-course measurements of caffeine and its metabolites extracted from fingertips after coffee intake: a preliminary study for the detection of drugs from fingerprints, Anal. Bioanal. Chem., 2013, 405(12), 39453952.

21 S. Muramoto, et al., Test Sample for the Spatially Resolved Quantification of Illicit Drugs on Fingerprints Using Imaging Mass Spectrometry, Anal. Chem., 2015, 87(10), 5444-5450.

22 G. Groeneveld, et al., Detection and mapping of illicit drugs and their metabolites in fingermarks by MALDI MS and compatibility with forensic techniques, Sci. Rep., 2015, 5, 11716.

23 E. Brunelle, et al., Step toward Roadside Sensing: Noninvasive Detection of a THC Metabolite from the Sweat Content of Fingerprints, ACS Sens., 2019, 4(12), 3318-3324.

24 M. Hudson, et al., Drug screening using the sweat of a fingerprint: lateral flow detection of $\Delta$ 9-tetrahydrocannabinol, cocaine, opiates and amphetamine, J. Anal. Toxicol., 2018, 43(2), 88-95.

25 S. Francese, et al., Beyond the ridge pattern: multi-informative analysis of latent fingermarks by MALDI mass spectrometry, Analyst, 2013, 138(15), 4215-4228.
26 D. R. Ifa, et al., Latent Fingerprint Chemical Imaging by Mass Spectrometry, Science, 2008, 321(5890), 805805.

27 F. Rowell, K. Hudson and J. Seviour, Detection of drugs and their metabolites in dusted latent fingermarks by mass spectrometry, Analyst, 2009, 134(4), 701-707.

28 S. J. Hinder and J. F. Watts, SIMS fingerprint analysis on organic substrates, Surf. Interface Anal., 2010, 42(6-7), 826829.

29 S. Bleay, et al., Fingerprint Source Book, ed. T. Kent, 2017, Home Office.

30 S. Sheraz, et al., Enhanced Ion Yields Using High Energy Water Cluster Beams for Secondary Ion Mass Spectrometry Analysis and Imaging, Anal. Chem., 2019, 91(14), 90589068.

31 S. Sheraz, et al., Prospect of increasing secondary ion yields in ToF-SIMS using water cluster primary ion beams, Surf. Interface Anal., 2014, 46(S1), 51-53.

32 J. S. Fletcher, et al., A New Dynamic in Mass Spectral Imaging of Single Biological Cells, Anal. Chem., 2008, 80(23), 9058-9064.

33 S. Sheraz née Rabbani, et al., Enhancing Ion Yields in Time-of-Flight-Secondary Ion Mass Spectrometry: A Comparative Study of Argon and Water Cluster Primary Beams, Anal. Chem., 2015, 87(4), 2367-2374.

34 M. C. Chambers, et al., A cross-platform toolkit for mass spectrometry and proteomics, Nat. Biotechnol., 2012, 30(10), 918-920.

35 A. M. Race, I. B. Styles and J. Bunch, Inclusive sharing of mass spectrometry imaging data requires a converter for all, J. Proteomics, 2012, 75(16), 5111-5112.

36 A. M. Race, et al., SpectralAnalysis: Software for the Masses, Anal. Chem., 2016, 88(19), 9451-9458.

37 M. J. Bailey, et al., Chemical Characterization of Latent Fingerprints by Matrix-Assisted Laser Desorption Ionization, Time-of-Flight Secondary Ion Mass Spectrometry, Mega Electron Volt Secondary Mass Spectrometry, Gas Chromatography/Mass Spectrometry, X-ray Photoelectron Spectroscopy, and Attenuated Total Reflection Fourier Transform Infrared Spectroscopic Imaging: An Intercomparison, Anal. Chem., 2012, 84(20), 8514-8523.

38 S. Sheraz née Rabbani, et al., Enhancing Secondary Ion Yields in Time of Flight-Secondary Ion Mass Spectrometry Using Water Cluster Primary Beams, Anal. Chem., 2013, 85(12), 5654-5658.

39 H. Reed, et al., The Reed-Stanton press rig for the generation of reproducible fingermarks: Towards a standardised methodology for fingermark research, Sci. Justice, 2016, 56(1), 9-17.

40 V. G. Sears, et al., A methodology for finger mark research, Sci. Justice, 2012, 52(3), 145-160. 